\title{
SIGNIFICADOS DO CONCEITO DE SAÚDE NA PERSPECTIVA DE DOCENTES DA ÁREA DA SAÚDE
}

\author{
Meanings of the health concept through the perspective of teachers in the health field \\ Significados del concepto de salud desde la perspectiva de los docentes del área de la \\ salud
}

Bárbara Brezolin Dalmolin

Diego Schaurich ${ }^{4}$

\author{
Dirce Stein Backes² \\ Juliana Silveira Colomé ${ }^{5}$
}

Cláudia Zamberlan ${ }^{3}$

Maria Helena Gehlen ${ }^{6}$

\section{RESUMO}

0 estudo objetivou compreender os significados do conceito de saúde para docentes profissionais da área da saúde de uma Instituição de Ensino Superior. Pesquisa descritivo-exploratória, realizada com os coordenadores dos cursos de graduação da área da saúde atuantes em atividades de ensino superior. Os resultados evidenciaram a existência de um paradoxo em relação aos significados atribuídos ao conceito de saúde. Para alguns, a saúde é entendida como um fenômeno complexo; para outros, um fenômeno reducionista. Os dados possibilitam argumentar que houve importantes avanços na apreensão teórica e conceitual de saúde, mesmo que para alguns participantes o fenômeno saúde ainda esteja pautado em concepções reducionistas e pontuais. Abarcar o significado ampliado do fenômeno saúde implica, em suma, ampliar as possibilidades interativas além da articulação teoria e prática, por meio de debates e discussões que for taleçam e divulguem o conceito de saúde segundo uma perspectiva ampliada.

Palavras-Chave: Educação em Saúde. Profissionais da Saúde. Pesquisa em Enfermagem.

\begin{abstract}
This study aimed to comprehend the meanings of the health concept for professional teachers of an Institution of Higher Education. This exploratory descriptive research was conducted with coordinators of undergraduate courses in the health field. The results demonstrated that there is a paradox related to the meanings attributed to the health concept. While some teachers understand health as a complex phenomenon, others see it as a reductionist phenomenon. The data suggest that there have been important advances in the theoretical and conceptual apprehension of health though for some participants the health phenomenon is still ruled by reductionist and punctual conceptions. Reaching the broader meaning of the health phenomenon implies not only enlarging the interactive possibilities, but also a theoretical and practical articulation through debates and discussions that strengthen and promote the concept of health according to a broader perspective.
\end{abstract}

Keywords: Health Education. Health Personnel. Nursing Research.

\section{Resumen}

El objetivo del estudio es comprender los significados del concepto de salud para docentes profesionales del área de la salud de una Institución de Enseñanza Superior. Investigación descriptivoexploratoria, realizada con los coordinadores de los cursos de licenciatura del área de la salud que trabajan en actividades de Enseñanza Superior. Los resultados evidencian la existencia de una paradoja en relación a los significados atribuidos al concepto de salud. Para algunos, la salud es entendida como un fenómeno complejo; para otros, un fenómeno reduccionista. Los datos permiten afirmar que ha habido importantes avances en la comprensión teórica y conceptual de la salud, mismo que para algunos de los participantes el fenómeno salud sea regido por la concepción reduccionista y puntual. Abarcar el significado del fenómeno ampliado de la salud implica, en definitiva, ampliar las posibilidades interactivas más allá de la articulación teoría y la práctica, a través de debates y discusiones que fortalezcan y revelen el concepto de salud de acuerdo con una perspectiva más amplia.

Palabras Clave: Educación en Salud. Personal de Salud. Investigación en Enfermería.

\footnotetext{
${ }^{1}$ Enfermeira. Graduada pelo Curso de Enfermagem do Centro Universitário Franciscano (UNIFRA). Santa Maria-RS. Brasil. E-mail: barbara_brezolin@hotmail.com. Enfermeira. Doutora em Enfermagem. Docente do Curso de Enfermagem do Centro Universitário Franciscano - UNIFRA. Líder do Grupo de Estudos e Pesquisa em Empreendedorismo Social da Enfermagem e Saúde (GEPESES/UNIFRA). Santa Maria-RS. Brasil. E-mail: backesdirce@ig.com.br. ${ }^{3}$ Enfermeira. Doutoranda em Enfermagem pelo Programa de Pós-Graduação em Enfermagem da Fundação Universidade Rio Grande - FURG. Docente do Curso de Enfermagem do Centro Universitário Franciscano - UNIFRA. Membro do Grupo Interdisciplinar de Pesquisa em Saúde (GIPES/UNIFRA). Santa Maria-RS. Brasil. E-mail: claudiazamberlanenator@gmail.com. ${ }^{4}$ Enfermeiro. Doutorando pelo Programa de Pós-Graduação da Escola de Enfermagem da Universidade Federal do Rio Grande do Sul (UFRGS). Santa Maria-RS. Brasil. E-mail: eu_diegosch@hotmail.com. ${ }^{5}$ Enfermeira. Mestre em Enfermagem. Docente do Curso de Enfermagem do Centro Universitário Franciscano - UNIFRA. Membro do Grupo Interdisciplinar em Pesquisa em Saúde (GIPES/UNIFRA). Santa Maria-RS. Brasil. E-mail: julianacolome@yahoo.com.br. ${ }^{6}$ Enfermeira. Mestre em Enfermagem. Docente do Curso de Enfermagem do Centro Universitário Franciscano - UNIFRA. Membro do Grupo de Estudos e Pesquisa em Empreendedorismo Social da Enfermagem e Saúde (GEPESES/UNIFRA). Santa Maria-RS. Brasil. E-mail: mahgehlen@terra.com.br.
} 


\section{INTRODUÇÃO}

A Organização Mundial da Saúde (OMS) conceitua saúde como um estado de completo bem-estar físico, mental e social e não apenas pela ausência de doenças ou enfermidades. ${ }^{1}$ Uma análise crítica e ampliada do conceito de saúde preconizado pela OMS permite delinear os seguintes questionamentos: é possível definir o que é completo bem-estar em uma sociedade marcada pelas injustiças e desigualdades sócio-econômicas crescentes? Como desenvolver um estado de completo bemestar no contexto socioeconômico na promoção da saúde? 0 que se entende atualmente por saúde ampliada?

Estudiosos, subsidiados pela crítica e pela reflexão, questionam o conceito de saúde como completo bem-estar e o consideram utópico, visto que esta ideia induz a uma condição de equilibrio, a qual é contraditória aos movimentos antagônicos que impulsionam novos modos de viver saudável, mesmo que em uma condição de doença. ${ }^{2-4}$

0 processo de viver com ou sem saúde não se reduz, portanto, a uma evidência orgânica, natural e objetiva e nem como um estado de equilibrio, mas está intimamente relacionada às características de cada contexto sociocultural e aos significados que cada indivíduo atribui ao seu processo de viver. As discussões que têm sido travadas em torno do processo saúde-doença têm gerado, sob um enfoque reducionista, a tendência natural de se pensar que a saúde significa a ausência de doença, ou seja, a saúde como sinônimo de evidências objetivas, associadas à ideia de que o corpo/físico está ou não funcionando adequadamente. ${ }^{5}$

A saúde, diferentemente do que muitos pensam, acreditam ou defendem, não pode ser apreendida como um fenômeno abstrato e nem mesmo como algo concreto ou atingível. Enquanto um fenômeno ampliado, a saúde envolve modos de ser e produzir e/ou recriar a vida em sua singularidade e multidimensionalidade. Nessa perspectiva, é preciso questionar os discursos que privilegiam o conceito de saúde somente pela sua dimensão biológica, assegurando uma concepção fragmentada do ser humano, bem como o caráter impositivo e normativo dos modos de se intervir na realidade dos indivíduos e comunidades. ${ }^{6}$

A partir do exposto, o processo saúde-doença depende, além das análises objetivas, da articulação com os diferentes determinantes da saúde, a fim de considerar tanto as evidências estruturais, isto é, os fatores externos do entorno social, quanto as condições de vida e de trabalho, as condições culturais, ambientais, entre outras. ${ }^{7}$

No campo denominado saúde, os profissionais, em especial a classe médica, centram-se, na maioria das vezes, em aspectos aparentes ou apenas nos sinais externos, reduzindo o ser humano a um objeto, desconsiderando-o em sua singularidade e historicidade enquanto sujeito da sua saúde. Tais práticas favorecem, mesmo com todos os avanços científicos, a reprodução fiel do modelo biotecnologicista, monopolizado pela medicina, no qual a comunicação socialmente relevante é a doença, ao invés da saúde. Essa concepção fragmentada e reducionista é, frequentemente, creditada pela indústria farmacêutica e tecnológica, a qual potencializa a doença ao invés de estimular o ser e o viver com saúde dos indivíduos. Desse modo, gera-se um aumento não racional de despesas para o sistema de saúde, além de monopolizar o mercado pela ênfase na doença. ${ }^{5}$

Não diferente dos sistemas privados, o Sistema Único de Saúde Brasileiro (SUS) reproduz, frequentemente, o modelo biomédico ao focar as práticas assistencialistas, ou seja, práticas focadas no modelo tradicional biologicista e determinista, em detrimento das ações integradas e integrativas voltadas para a promoção e educação da saúde. Tais considerações requerem novos debates e discussões, a fim de ampliar o conceito de saúde, pela valorização do ser humano como um ser uno e multidimensional, isto é, capaz de manter-se saudável ou viver com saúde, mesmo que em uma condição de doença.

Deste processo de debates e discussões, também os docentes, profissionais de saúde que atuam na área do ensino, formadores de opiniões em saúde, devem fazer parte da construção de novos referenciais que possibilitem um conceito ampliado de saúde, apreendido enquanto um fenômeno integral, integrador e potencializador de um viver com mais saúde. Nessa direção, têm-se como questões de pesquisa: qual o significado do conceito de saúde para os docentes profissionais da área da saúde de uma Instituição de Ensino Superior?

Neste sentido, o objetivo delineado para este estudo foi compreender os significados do conceito de saúde para docentes profissionais da área da saúde de uma Instituição de Ensino Superior.

\section{METODOLOGIA}

Caracteriza-se como uma pesquisa descritivoexploratória. No contexto da metodologia qualitativa aplicada à saúde, emprega-se a concepção trazida das Ciências Humanas, segundo as quais não se busca estudar o fenômeno em si, mas entender seu significado individual ou coletivo para a vida das pessoas. ${ }^{9}$

A pesquisa foi realizada em uma Instituição de Ensino Superior localizada na região central do Estado do Rio Grande do Sul. Esta Instituição mantém atualmente em funcionamento 31 cursos de graduação, sendo sete da Área da Saúde: Biomedicina, Enfermagem, Farmácia, Fisioterapia, Nutrição, Odontologia e Terapia Ocupacional.

Fizeram parte da amostra docentes profissionais dos diferentes cursos de graduação da área da saúde, mais especificamente os coordenadores dos cursos que também atuaram em atividades de ensino por ocasião da realização deste estudo. Para tanto, foram entrevistados coordenadores dos Cursos de Enfermagem, Fisioterapia, Nutrição, Farmácia, Biomedicina, Odontologia, Terapia Ocupacional 
e do Curso Técnico de Enfermagem, totalizando oito participantes.

Os dados foram coletados por meio de uma entrevista com questões norteadoras, tais como: o que significa saúde para você? Que elementos compõem o fenômeno saúde? Qual a compreensão de saúde predominante no contexto socioeconômico atual? 0 que envolve o conceito de saúde? As mesmas foram realizadas em dias e horários previamente agendados, entre os meses de junho a setembro de 2009. Todas as entrevistas foram gravadas e, a seguir, transcritas.

Os dados foram analisados por categorização, com base no método de análise de conteúdo, ${ }^{10}$ o qual se constitui de três momentos: o primeiro consiste em uma frequência com identificação das principais percepções dos docentes entrevistados; o segundo analisa o conteúdo que identifica as categorias que emergiram a partir dos dados coletados; e o terceiro refere-se à interpretação das categorias, compreendendo suas significações.
Quanto aos critérios éticos, foram atendidas as recomendações da Resolução do Conselho Nacional de Saúde CNS n 196/96, que prescreve a ética na pesquisa com seres humanos. ${ }^{11} 0$ projeto de pesquisa foi aprovado pelo Comitê de Ética da Instituição onde a pesquisa foi realizada e encontra-se registrado sob o número 168/08.

\section{RESULTADOS}

Os dados analisados resultaram em duas categorias: saúde, como um conceito amplo e complexo, e saúde, como um fenômeno reducionista. Na sequência, será apresentado o esquema que integra as categorias ao tema central: evidenciando um paradoxo entre o conceito ampliado e 0 conceito reducionista de saúde. Desse modo, contrastam os códigos visão assistencialista, concepção curativista, medicalização da saúde, práticas pontuais, foco na doença, completo bem-estar, singularidade, promoção da saúde, multidisciplinaridade e humanização, formando um processo circular e integrativo

Figura 1: Evidenciando um paradoxo entre os conceitos de saúde de coordenadores dos Cursos da Área da Saúde. Santa Maria, RS, 2009.
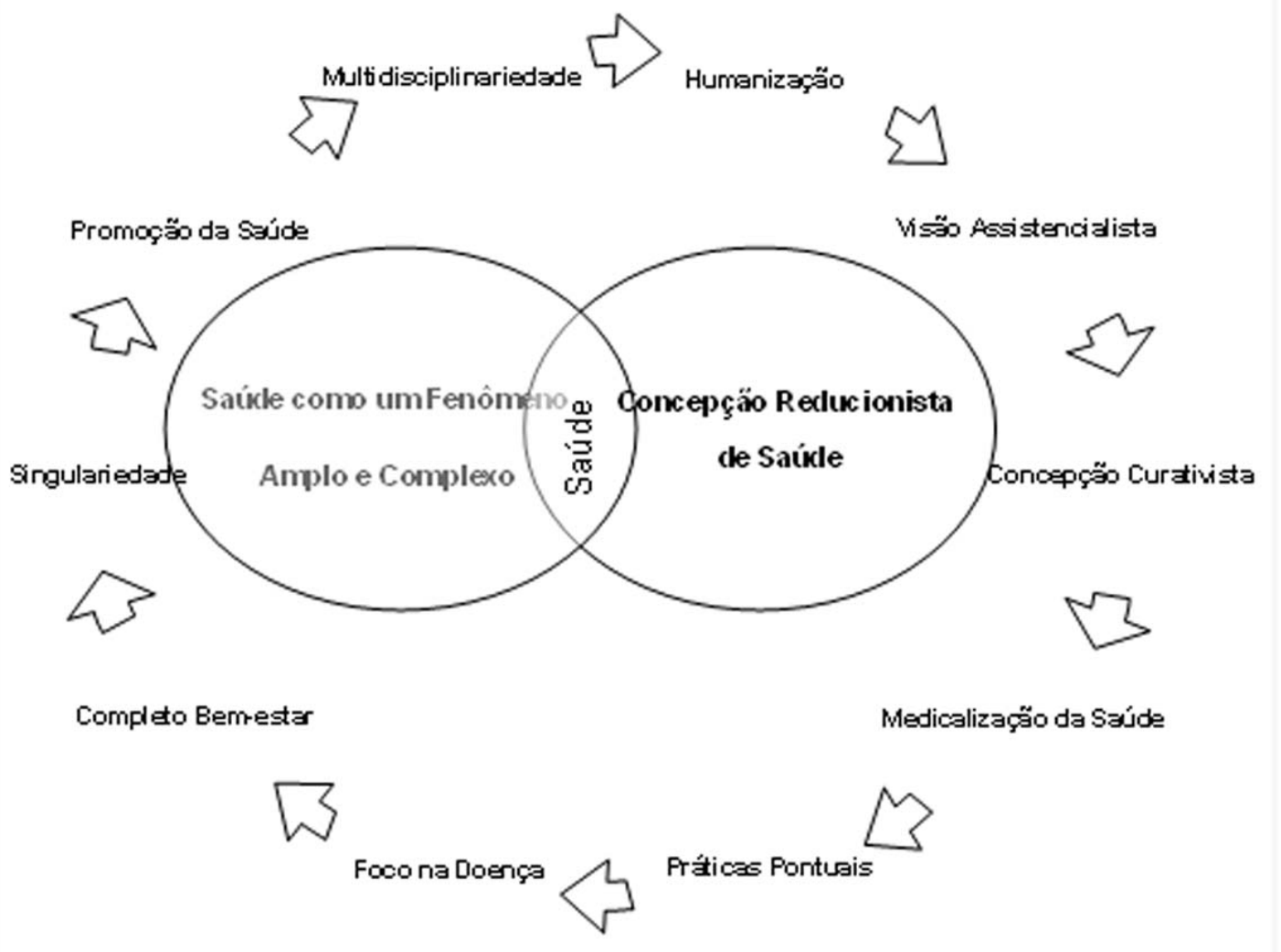

0 tema central: evidenciando um paradoxo entre os conceitos de saúde de coordenadores dos Cursos da Área da Saúde, conforme expresso na Figura 1, possibilita visualizar as interconexões entre ambos os conceitos, os quais se interligam e, por vezes, se complementam e se confundem no processo saúde-doença.

A categoria saúde como um fenômeno amplo e complexo, evidencia que já existe um esforço por parte de alguns profissionais no sentido de ampliar o conceito de saúde em relação aos determinantes da saúde. Isso fica evidente quando acenam para a promoção da saúde, a integralidade, a singularidade, a humanização, a multidisciplinaridade, a 


\section{Significados do conceito de saúde para docentes}

interdisciplinaridade e o completo bem-estar, considerando, também, a importância do diálogo, da escuta, do carinho, do amor e da qualidade de vida.

As falas a seguir refletem o conceito ampliado de saúde:

Na verdade, saúde não significa simplesmente a ausência de doença. Saúde tem um amplo espectro que envolve a condição socioeconômica, o saneamento básico, as condições psíquicas, emprego... Então, uma condição de equilibrio entre todas essas parcelas é que são importantes na vida das pessoas (Margarida).

Eu acredito que saúde, hoje, não é somente a ausência de doença. A saúde envolve aspectos físicos, psíquicos, sociais, ambientais, culturais, muito mais amplo do que antigamente a gente pensava (Violeta).

Da mesma forma como a saúde, o cuidado em saúde foi evidenciado como um fenômeno amplo e complexo, motivado pelas interações entre a escuta e o diálogo, como refletem as falas a seguir:

O cuidar em saúde eu acho muito complexo, porque quando a gente cuida de alguém a gente não cuida só da doença, não somente da patologia, a gente vai cuidar de um todo, do ser integral, psíquico, fisiológico, emocional, com amor e carinho. Eu sempre digo que a gente tem que cuidar como a gente gostaria de ser cuidado, envolvendo todos os aspectos pra realizar um cuidado de excelência (Amor-Perfeito).

Para mim, cuidado em saúde tem a ver também com prevenção e promoção, antes da reabilitação. Para chegar lá no nivel mais alto de complexidade, acredito que primeiro tenha que pensar nos cuidados primários, como a prevenção e a promoção, para evitar então que se alastre para um nível mais superior de complexidade (Crisântemo).

A categoria saúde como um fenômeno reducionista demonstra que apesar dos avanços na área da saúde, persiste, em alguns casos, a concepção reducionista e linear no discurso de profissionais. Este enfoque é evidenciado por meio de ações pontuais, assistencialistas e curativistas de promover e intervir no binômio saúde-doença, de acordo com as falas a seguir:

A nossa população atual e no nosso caso também da (...) e de qualquer outra área, as pessoas gostam muito de falar em prevenção, mas as pessoas não podem esquecer que elas já estão doentes (Tulipa).
Observa-se que, para muitos profissionais, o conceito de saúde não parece estar bem definido, uma vez que a maioria dos participantes focou a saúde como ausência de doença, a partir de um olhar reducionista, curativista e assistencialista.

\section{DISCUSSÕES}

0 conceito de saúde, na forma como foi evidenciado nas falas dos participantes, acena para a necessidade de se ampliar os debates sobre a sua conceituação e a configuração teórica atual sobre o sistema de saúde, mais especificamente no SUS.

Na VIII Conferência Nacional de Saúde, grandes avanços favoreceram a base conceitual de saúde, graças à participação ativa da sociedade, a qual impulsionou o Movimento da Reforma Sanitária, que possibilitou a reformulação do sistema de saúde brasileiro, bem como o entendimento de saúde enquanto produto de múltiplos determinantes, como educação, trabalho, alimentação, acesso aos serviços de saúde, dentre outros. Com o surgimento da Reforma Sanitária, a saúde passou a ser vista de forma ampla e complexa, centrando as diretrizes de universalidade, integralidade e equidade, diferente do modelo de saúde anterior, que evidenciava um sistema fragmentado, assistencialista, voltado para a lógica curativista, sem a participação da população e restrito aos profissionais da saúde. ${ }^{11-12}$

0 Movimento da Reforma Sanitária foi uma resposta à crise em que o país se encontrava, em especial às crises de conhecimentos e de modelo biomédico, de autoritarismo, das condições sanitárias da população e do sistema de prestação de serviços à saúde. Por meio destas condições problemáticas, o governo ampliou os debates acerca do processo saúdedoença ${ }^{12}$, suscitando nos profissionais da saúde a necessidade de ampliar estes conceitos e inovar as práticas de cuidado em saúde.

O SUS, institucionalizado pela Lei Orgânica de Saúde 8.080 de 1990, preconiza que todo o cidadão brasileiro tenha acesso aos serviços de saúde. Logo, é um sistema de saúde altamente empreendedor, pela ampla cobertura social e cujo foco passa a centrar-se na educação, promoção e proteção da saúde. ${ }^{14}$

Nessa perspectiva, as políticas de promoção e proteção da saúde regem os serviços prestados pelo SUS aliando a participação popular nas decisões que dizem respeito ao vivermelhor ou viver com saúde. Fundamentado em princípios ideológicos e doutrinários, quais sejam: a descentralização, a regionalização e a hierarquização e a participação popular, 0 SUS se configura como um dos sistemas mais reconhecidos e resolutivos, mesmo que ainda longe de se concretizar plenamente. ${ }^{13-14}$

O SUS preconiza que todos tenham direito, quando necessário, aos serviços de saúde, de forma que as carências da sociedade, em especial as emergentes, sejam atendidas 
com acesso a todas as esferas de serviços, sem discriminação. É preciso, no entanto, que essas carências sejam revisitadas da forma mais ampla possível, abrangendo os aspectos biopsicossociais, espirituais, culturais, avaliando a contextualização em que esse indivíduo está inserido.

No âmbito deste estudo, entretanto, se evidencia um paradoxo entre o conceito de saúde, entendido como um fenômeno complexo e, de outro modo, a saúde como um fenômeno reducionista. É preciso, nessa direção, repensar o ser e fazer na área da docência, a fim de atender os princípios do sistema de saúde vigente, bem como instigar novos referenciais capazes de repensar a saúde para além da dimensão biológica. Superar a ideia objetiva e assistencialista do conceito de saúde implica abarcar a integralidade do cuidado em saúde pela apreensão do ser humano como um ser integral e complexo, isto é, inserido em um contexto social contraditório. ${ }^{15}$

É inegável que a Reforma Sanitária possibilitou grandes avanços no que se refere à ampliação do conceito de saúde, do ser humano, apreendido em seu contexto real e concreto. Também proporcionou avanços pelo desenvolvimento de novos valores profissionais e a criação de instrumentos de gestão democráticos, bem como o favorecimento ao controle social. ${ }^{12}$

Igualmente, a VIII Conferência Nacional de Saúde resultou em mudanças significativas na saúde pública. Observase, porém, que ao longo dos 20 anos de SUS, muitos profissionais não conseguiram ampliar a concepção do conceito de saúde, conforme evidenciam os resultados anteriormente apresentados. Ao permear a denominação reducionista de saúde, muitos profissionais ainda privilegiam a etiologia biologicista ao enfocar a concepção fragmentada e curativista, sem avaliar os aspectos sociais, psicológicos, ambientais, culturais e ecológicos em que vive e se encontra a sociedade. ${ }^{16}$

Para alguns participantes do estudo, o conceito de saúde parece estar associado a um olhar biologicista, reduzindo-se apenas ao processo de cura, deixando de lado os determinantes sociais, psicológicos, espirituais e contextuais do indivíduo. ${ }^{17}$ Percebe-se, portanto, que apesar dos grandes avanços e conquistas no processo de saúde brasileiro, ainda perdura, por parte de alguns profissionais da saúde, a abordagem ao modelo biologicista, mecanicista e materialista, a qual se restringe ao cuidado técnico, aos aspectos do adoecimento, desconsiderando, muitas vezes, o usuário em sua integralidade e na sua dimensão singular, postando, com isso, a humanização do cuidado em segundo plano. ${ }^{18}$

Embora ainda existam profissionais que supostamente não aderiram à nova concepção de saúde, como explana 0 modelo de saúde brasileiro, há por outro lado profissionais que conseguiram adotar esta nova percepção de saúde, conforme evidenciam as falas dos participantes. Cabe ao profissional da saúde fazer valer e se guiar por esse sistema de saúde tão abrangente como o SUS.

Os resultados demonstraram, em suma, a existência de um paradoxo em relação aos significados atribuídos ao conceito de saúde. Para alguns, este é entendido como um fenômeno complexo e para outros como um fenômeno reducionista, os quais permitem concluir que existe a necessidade de instigar novos referenciais capazes de repensar e ampliar o conceito de saúde para além da dimensão técnica e curativista.

\section{CONSIDERAÇÕES FINAIS}

Compreender os significados que os profissionais da área da saúde atribuem ao fenômeno saúde possibilita argumentar que houve importantes avanços na apreensão teórica e conceitual de saúde, mesmo que para alguns participantes o fenômeno saúde ainda esteja pautado em concepç̃̃es reducionistas e pontuais.

Nessa perspectiva, há uma necessidade de os sujeitos da pesquisa ampliarem o conceito de saúde transcendendo o cenário de formação acadêmica, com o olhar centrado para a humanização e para a escuta, observando o indivíduo na sua singularidade e no contexto real em que vive.

Assim, as políticas públicas deverão ser capazes de qualificar as práticas educativas e motivacionais dos profissionais da saúde por meio de estratégias que considerem os significados que os indivíduos, famílias e comunidades atribuem ao processo saúde-doença. É necessária, também, a operacionalização de iniciativas empreendedoras por parte dos entrevistados, as quais estejam voltadas para a promoção, proteção e educação da saúde nos diferentes cenários sociais e ambientais.

Abarcar o significado ampliado do fenômeno saúde implica, em suma, ampliar as possibilidades interativas além da articulação teoria e prática, por meio de debates e discussões que fortaleçam e divulguem o conceito de saúde segundo uma perspectiva ampliada. Para tanto, torna-se fundamental, ainda, compreender o ser humano como um ser singular e multidimensional, que está inserido em um contexto real e concreto.

\section{REFERÊNCIAS}

1 Organização Mundial da Saúde- OMS. Carta da Organização Mundial de Saúde, 1946.[citado 2009 out 18]. Disponível em http:// www.onuportugal.pt/oms.doc.

2 Andrade SR. Gestão das práticas de saúde na perspectiva do cuidado complexo. Texto\& Contexto Enferm. 2006 jul/set; 15(3): 483-91.

3 Calazans R, Lustoza RZ. A medicalização do psíquico: os conceitos de vida e saúde. Arq Bras Psicol. 2008 jan/jun; 60(1): 124-31.

4 Menicucci TMG. 0 Sistema Único de Saúde- 20 anos: balanço e perspectivas. Cad Saude Publica. 2009 jul; 25(7): 1620-25. 


\section{Significados do conceito de saúde para docentes}

5 Yépez MAT, Pinheiro VS. Adolescência, saúde e contexto social: esclarecendo práticas. Psicol Soc. 2002 jul/dez; 14(2): 133-47.

6 Bernardes AG, Guareschi NMF, Medeiros PF. 0 conceito de saúde e suas implicações nas práticas psicológicas. Psicol: Teoria Pesq. 2005 set/ dez; 21(3): 263-9.

7 Reis J. Modelo metateórico da psicologia da saúde para o século XXI: interação ou integração biopsicossocial? Rev Analise Psicol. 1999; 3(17): 415-33.

8 Turato ER. Tratado da metodologia da pesquisa clínico-qualitativa: construção teórico-epistemológica, discussão comparada e aplicação nas áreas da saúde e humanas. Petrópolis(RJ): Vozes; 2003.

9 Bardin L. Análise de conteúdo. $4^{\mathrm{a}}$ ed. Lisboa: Ed 70; 2009.

10 Ministério da Saúde (BR). Conselho Nacional de Saúde. Resolução n¹96, de 10 de outubro de 1996. Diretrizes e normas regulamentadoras de pesquisa envolvendo seres humanos. Brasília (DF); 1997.

11 Ministério da Saúde (BR). Anais da 8 a Conferência Nacional de Saúde. Brasília(DF) 1986. [citado 2009 out 18] Disponível em: http:// portal.saude.gov.br/portal/saude/cidadao.

12 Fleury S. Reforma sanitária brasileira: dilemas entre o instituinte e o instituído. Cienc Saude Colet. 2009 maio/jun; 14(3): 743-52.

\section{NOTAS}

Participaram ainda da construção deste artigo as autoras: Regina Santini Costenaro. Enfermeira. Doutora em Enfermagem. Docente do Curso de Enfermagem da UNFRA. Líder do Grupo Interdisciplinar de Pesquisa em Saúde - GIPES/UNIFRA. Santa Maria, Rio Grande do Sul, Brasil.E-mail: regina@unifra.br.

Simone dos Santos Nunes. Enfermeira. Mestranda em Geomática. Docente do Curso de Enfermagem da UNFRA. Membro do Grupo Interdisciplinar de Pesquisa em Saúde - GIPES/UNIFRA. Santa Maria, Rio Grande do Sul, Brasil.E-mail: simonenunes@yahoo.com.br
13 Ministério da Saúde (BR). Lei Orgânica 8080 de 1990. [ citado 2009 out 18 ]. Disponível em http://200.214.130.38/portal/arquivos/pdf/ Lei8080.pdf.

14 Backes DS, Koerich MS, Rodrigues ACRL, Drago LC, Klock P, et al. 0 que os usuários pensam e falam do Sistema Único de Saúde? Uma análise dos significados à luz da Carta dos Direitos dos Usuários. Cien Saude Colet. 2009 maio/jun, 14(3): 903-10.

15 Backes DS. Vislumbrando o cuidado de enfermagem como prática social empreendedora [tese]. Florianópolis: Centro de Ciências da Saúde, Universidade Federal de Santa Catarina; 2008.

16 Yépez MT. A interface psicológica social e saúde: perspectivas e desafios. Psicol Est. 2001 jul/dez; 6(2): 49-56.

17 Rizzoto MLF. A origem da enfermagem profissional no Brasil: determinantes históricos e conjurais. [citado 2009 nov 01]. Disponível em: http://www.histedbr.fae.unicamp.br/navegando/artigos_frames/ artigo_077.html.

18 Erdmann AL, Nascimento KC, Marcelino G, Ribeiro JA. As interfaces do cuidado pelo olhar da complexidade: um estudo com um grupo de pósgraduandos de enfermagem. Esc Anna Nery. 2005 dez; 9(3): 411-20. 